\title{
Synchronous Chemoradiotherapy in Patients with Stage III and IV Head and Neck Cancer: Comparing Cisplatin with Capecitabine*
}

\author{
Sherif A. Raafat ${ }^{1}$, Emmad E. Habib ${ }^{2}$, Ashraf M. Maurice ${ }^{2}$ \\ ${ }^{1}$ Otolaryngology Department, Faculty of Medicine, Cairo University, Cairo, Egypt; ${ }^{2}$ Clinical Oncology Department, Faculty of \\ Medicine, Cairo University, Cairo, Egypt. \\ Email: e.habib@kasralainy.edu.eg
}

Received September 20 ${ }^{\text {th }}$, 2012; revised October 21 ${ }^{\text {st }}$ 2012; accepted October 31 ${ }^{\text {st }}, 2012$

\begin{abstract}
Purpose: To evaluate the efficacy of concurrent intravenous cisplatin versus oral capecitabine with radical radiotherapy in locally advanced squamous cell carcinoma of the head and neck. Materials and Methods: Between January 2007 and December 2009, 60 patients with stage III/IV head and neck squamous cell carcinoma (0 to 1 performance status) were enrolled into this study. Thirty cases are given cisplatin $30 \mathrm{mg} / \mathrm{m}^{2} \mathrm{IV}$ infusion weekly for 6 weeks with conventional radiotherapy. The remaining thirty cases are given oral capecitabine $500 \mathrm{mg} / \mathrm{m}^{2}$ twice daily, continuously for 28 35 days with conventional radiotherapy also. The radiotherapy dose was 4600 cGy in 20 fractions over 4 weeks to primary and neck nodes followed by boost to primary site and any residual disease 1500 - 2000 cGy in 6 to 8 fractions. Results: The median age was 53 (range 25 - 71) years; 10 cases had stage III disease, 36 cases IVa disease and 14 cases IVb disease. Seventy-three percent of patients completed the course of capecitabine and $80 \%$ completed prescribed cisplatin. There were no treatment-related deaths, grade 4 haematological toxicity or grade 3 renal toxicity in either arm. The complete response rate at 3 months was $77 \%$ (23/30 patients) in the capecitabine group and 60\% (18/30) in the cisplatin group. Relapse occurred in 10/30 (33\%) patients by 2 years in the capecitabine group and in 12/30 (40\%) in the cisplatin group. On analysis of survival data, the median follow-up period was $35 \pm 15$ months for overall survival and $33 \pm 10$ months for disease free survival. The overall survival, and disease-free survival rates at 2 years were $67 \%$, and $85 \%$, respectively for the capecitabine group versus $60 \%$ and $73 \%$ for the cisplatin group. Conclusion: Synchronous chemo-radiotherapy with capecitabine was found to be very effective, with excellent response, local control and 3-year cancer-specific survival rates.
\end{abstract}

Keywords: Capecitabine; Cisplatin; Advanced Squamous Cell Carcinoma of the Head and Neck; Synchronous Chemoradiotherapy

\section{Introduction}

Worldwide, more than 650,000 patients are diagnosed with squamous cell carcinoma of the head and neck (SCCHN) yearly, accounting for $6 \%$ of all cancers [1,2]. Two-thirds of the SCCHN are in a loco-regionally advanced stage at diagnosis.

Chemo-radiotherapy is regarded as a standard treatment for stage III and IV squamous cell carcinoma of the head and neck. Timing of the chemotherapy has for long been a matter of debate but concurrent chemo-radiation was widely adopted as standard of care for locally advanced squamous cell carcinoma of the head and neck after the publication of a large meta-analysis which demonstrated that concurrent chemo-radiation confers an

* Conflict of interest statement: None declared. absolute survival benefit of $8 \%$ at 2 and 5 years [3,4]. Cisplatin given weekly has been widely reported [4]. However, there remain concerns that adding chemotherapy to radiotherapy increases both acute and late sideeffects. Studies using chemo-radiotherapy, have reported a treatment-related mortality up to $5 \%$, which patients and oncologists would find unacceptable [5,6]. Adding chemotherapy to radiotherapy also leads to problems with chronic dysphagia with long-term Ryle tube feeding in $10 \%-30 \%$ of patients $[7,8]$. Even if biological targeting agents are eventually confirmed to produce a clearer improvement in therapeutic gain [9], there remains, for now, a need to explore synchronous chemo-radiotherapy using agents other than cisplatin to reduce toxicity, widen applicability and reduce financial burden of treatment.

Cisplatin, or cis-diamminedichloroplatinum was first 
described by Michele Peyrone in 1845, and known for a long time as Peyrone's salt [10]. The structure was deduced by Alfred Werner in 1893 [11]. In 1965, Barnett Rosenberg, van Camp et al. of Michigan State University discovered that electrolysis of platinum electrodes generated a soluble platinum complex which inhibited binary fission in Escherichia coli bacteria [12]. Cisplatin was approved for use by the US. Food and Drug Administration on December 19, 1978 [13]. Note that although cisplatin is frequently designated as an alkylating agent, it has no alkyl group. It is correctly classified as alkylatinglike.

On the other hand, we chose to study capecitabine because it is a tumour activated fluoro-pyrimidine building on the proven radio-sensitizing track record of 5fluorouracil [14] and oral administration should permit flexibility and promote patient acceptability and limit time spent in the hospital. The activation of capecitabine follows a pathway with three enzymatic steps and two intermediary metabolites, 5'-deoxy-5-fluorocytidine (5'DFCR) and 5'-deoxy-5-fluorouridine (5'-DFUR), eventually forming 5-fluorouracil [15]. Side-effects of capecetabine are individual and may include one or more of the following. Hand-foot syndrome which can lead to the disappearance of fingerprints. Diarrhea, nausea, and stomatitis have occurred. Neutropenia, anemia, thrombocytopenia and hyper-billirubinaemia [16].

Capecitabine may interact with warfarin and increase bleeding risk and may inhibit cytochrome CYP2C9 enzyme, and therefore increase levels of substrates such as phenytoin [16].

\subsection{Manufacturer's Warning}

Leucovorin increased the toxicity of capecitabine without any apparent advantage in response rate.

\subsection{Aim of Work}

To evaluate the efficacy, acceptability and tolerability of concurrent intravenous cisplatin versus oral capecitabine with radical radiotherapy in locally advanced squamous cell carcinoma of the head and neck.

\subsection{Rationale}

Based on the study performed by Sykes A. J., et al. Results of a phase I study to determine the maximum tolerated dose of capecitabine when given concurrently with radical radiotherapy in the treatment of squamous cell carcinoma of the head and neck [15]. We applied these findings to our study design. In the target group of stage III/IV head and neck squamous cell carcinoma cases, the response rate using radiotherapy alone is about $70 \%$. It was anticipated that the response rate would be somewhat higher with the addition of capecitabine. This anticipation was based on previously published reports on cisplatin with radiotherapy.

\section{Materials and Methods}

Between January 2007 and December 2009, 60 patients with stage III/IV head and neck squamous cell carcinoma (0 to 1 performance status) were enrolled into this study. Inclusion Criteria:

- Stage III/IV head and neck pathologically proven squamous cell carcinoma diagnosed by biopsy only.

- 0 to 1 WHO performance status.

- Patients with normal blood counts and blood chemistry.

- Inoperable cases with invasion of vital nearby structures.

- Advanced cases refusing mutilating surgery.

Exclusion Criteria:

- Tumours of the nasopharynx, and salivary glands were excluded.

- Patients who received radio or chemotherapy previously.

- Confirmed radiologically and by isotopic bone scanning the presence of hematogenous distant metastases.

- The presence of any debilitating disease as diabetes, heart disease, liver or kidney impairment.

- The presence of concomitant auto-immune disease.

- Cases with double malignancy.

The study was conducted after approval by the ethical review board. Consent was obtained from each patient before entering the study. Recommendations of the Declaration of Helsinki for biomedical research involving human patients were also followed. A previous study performed by Sykes A. J., et al. (Results of a phase I study to determine the maximum tolerated dose of capecitabine when given concurrently with radical radiotherapy in the treatment of squamous cell carcinoma of the head and neck) had also obtained a similar previous approval by the appropriate ethical review boards [15]

Thirty cases are given cisplatin $30 \mathrm{mg} / \mathrm{m}^{2}$ IV infusion weekly for 6 weeks with conventional radiotherapy. The remaining thirty cases are given oral capecitabine 500 $\mathrm{mg} / \mathrm{m}^{2}$ twice daily, continuously for 28 - 35 days with conventional radiotherapy also. The tablets could be crushed and delivered with liquids in case of dysphagia or Ryle tube feeding. The taking of capecitabine was not linked chronologically to the radiotherapy appointment time. All patients were reviewed in the weekly "Reaction Clinic" with assessment of acute toxicities, weight, blood counts and biochemical profile. Acute and late side-ef- 
fects were scored using the WHO scoring system. All patients were treated supine, neck neutral and with immobilization in a thermoplastic shell. Treatment was planned using two-dimensional simulation using a lateral parallel opposed pair arrangement of fields with lower neck nodes treated with a separate field with midline shielding. The radiotherapy dose was as follows; phase I: 4600 cGy in 20 fractions over 4 weeks followed by; phase II: boost to primary site and any residual disease excluding the spinal cord delivering a dose of 1500 2000 cGy in 6 to 8 fractions.

We evaluated the overall response rate (complete and partial), toxicity, loco-regional control, overall survival, and disease free survival.

Follow-up was continued monthly during the first year, 2 monthly for the second year. At each follow-up visit, a history was taken and a clinical examination, including laryngoscopy, was carried out. The late effects were recorded after 1 year.

Study end points were the date of last follow-up, death, disease progression, or persistent treatment toxicity resulting in intolerance to treatment and changing the line of treatment.

Criteria of response were as follows:

- Complete response (CR): complete disappearance of disease.

- Partial response (PR): 50\% - 90\% decrease in tumour volume.

- Stationary Disease (SD): $<50 \%$ decrease in tumour volume.

- No response (NR): no change in the size of the tumour.

- Disease Progression (DP): increase in the size of the tumor.

\section{Statistical Methods}

A phase II randomized trial of concomitant chemo-radiotherapy in patients with Locally Advanced Squamous Cell Carcinoma of the Head and Neck.

Data was analyzed using SPSSwin statistical package version 15 (SPSS Inc., Chicago, IL). Chi-square test (Fisher's exact test) was used to examine the relation between qualitative variables. For quantitative data, comparison between the two arms was done using independent sample t-test or Mann-Whitney test. Survival analysis was done using Kaplan-Meier method and comparison between two survival curves was done using log-rank test. A p-value $<0.05$ was considered significant.

\section{Results}

The patient characteristics are summarized in Table 1.
Table 1. Patient characteristics.

\begin{tabular}{|c|c|c|c|c|}
\hline Group & \multicolumn{2}{|c|}{ Capcetabine } & \multicolumn{2}{|c|}{ Cisplatin } \\
\hline Age & $25-71$ & $\begin{array}{c}\text { Median } \pm \text { SD } \\
49 \pm 11.8\end{array}$ & $25-68$ & $\begin{array}{c}\text { Median } \pm \text { SD } \\
56.8 \pm 10.8\end{array}$ \\
\hline Males to Females & $22: 8$ & & $16: 14$ & \\
\hline Smokers & 15 & $50 \%$ & 12 & $40 \%$ \\
\hline Alcohol Intake & 3 & $10 \%$ & 1 & $3.3 \%$ \\
\hline \multicolumn{5}{|l|}{ Performance Status: } \\
\hline 0 & 16 & $53 \%$ & 16 & $53 \%$ \\
\hline 1 & 14 & $47 \%$ & 14 & $47 \%$ \\
\hline \multicolumn{5}{|l|}{ Site of Disease: } \\
\hline Nasal Cavity & 1 & $3.3 \%$ & 0 & 0 \\
\hline Paranasal Sinus & 4 & $13 \%$ & 6 & $20 \%$ \\
\hline Oral Cavity & 3 & $10 \%$ & 7 & $23 \%$ \\
\hline Oropharynx & 1 & $3.3 \%$ & 3 & $10 \%$ \\
\hline Larynx & 13 & $43 \%$ & 11 & $37 \%$ \\
\hline Hypopharynx & 8 & $27 \%$ & 3 & $10 \%$ \\
\hline \multicolumn{5}{|l|}{ Grade: } \\
\hline 1 & 0 & 0 & 2 & $7 \%$ \\
\hline 2 & 17 & $57 \%$ & 9 & $30 \%$ \\
\hline 3 & 7 & $23 \%$ & 10 & $33 \%$ \\
\hline Undifferentiated & 6 & $20 \%$ & 9 & $30 \%$ \\
\hline \multicolumn{5}{|l|}{ Stage } \\
\hline III & 5 & $17 \%$ & 5 & $17 \%$ \\
\hline IVA & 20 & $67 \%$ & 16 & $53 \%$ \\
\hline IVB & 5 & $17 \%$ & 9 & $30 \%$ \\
\hline
\end{tabular}

The response rate is summarized in Figure 1.

The complete response rate at 3 months was $77 \%$ (23/ 30 patients) and the partial responses were 13\% (4/30), stationary disease occurred in 2 cases and progressive disease in 1 case in the capecitabine group. Whereas, the complete responses were $60 \%(18 / 30)$ and the partial responses $23 \%$ (7/30), stationary disease was found in 3 cases and progressive disease in 2 cases in the cisplatin group. Persistent disease at 3 months was recorded as treatment failure. Relapse occurred in 10/30 (33\%) patients by 1 year in the capecitabine group and in 12/30 $(40 \%)$ in the cisplatin group.

Eleven (33\%) patients required Ryle tube feeding in the capecetabine group and $15(50 \%)$ in the cisplatin group.

All patients finished their radiotherapy, but there were 
delays in treatment because of mucositis. Delays ranged from 3 - 7 days in the capecitabine group and 2 - 5 days in the cisplatin group and the lag period dose was calculated and added.

Capecitabine was stopped after 4 weeks of administration in 5 cases, and after 5 weeks of administration in 3 cases because of diarrhea and palmoplantar erythema.

Cisplatin was stopped after 4 weeks in 6 cases because of impaired renal function.

The rest of the cases completed their prescribed chemotherapy.

Acute toxicity is summarized in Table 2.

There was no grade 4 haematological toxicity. A fall in the haemoglobin level was seen in 11/30 (37\%) in the capecitabine group and in 2/30 (7\%) in the cisplatin group. Grade 1 neutropenia was seen in 4/30 (13\%) and $3 / 30(10 \%)$ in the capecitabine group and cisplatin group respectively. Moreover, grade 2 neutropenia was detected in 8/30 (27\%) and 1/30 (3.3\%) in the capecitabine group and cisplatin group respectively. Only in the capecitabine group grade 3 neutropenia was noted in 5/30 (17\%) of the cases necessitating temporary treatment stoppage.

Other systemic toxicity included grade $1 / 2$ nausea/ vomiting which was found in 18 cases (60\%) for nausea and in 19 cases $(63 \%)$ for vomitting in the capecitabine group. Grade 3 nausea/vomiting in the capecitabine group was rare 1 case only with nausea and 1 case with vomiting. For the cisplatin group grade $1 / 2$ nausea/vomiting was found in 8 cases (27\%) for nausea and in 8 cases (27\%) for vomiting. Grade 3 nausea/vomiting in the cisplatin group was rare 1 case only with nausea and
1 case with vomiting. All the cases responded well to anti-emetic therapy (P value 0.01 ).

Grade 1/2 diarrhea was noticed only in the capecitabine group in 4 cases (13\%) and grade 3 in 1 case and was controlled with anti-diarrheal medication. Palmoplantar syndrome developed only in the capecitabine group in 3 cases (10\%) and was managed by temporary stopping the drug for 3 - 5 days and treatment with topical steroids.

Alopecia outside the radiation field was more marked in the capecitabine group. Grade $1 / 2$ was found in 29

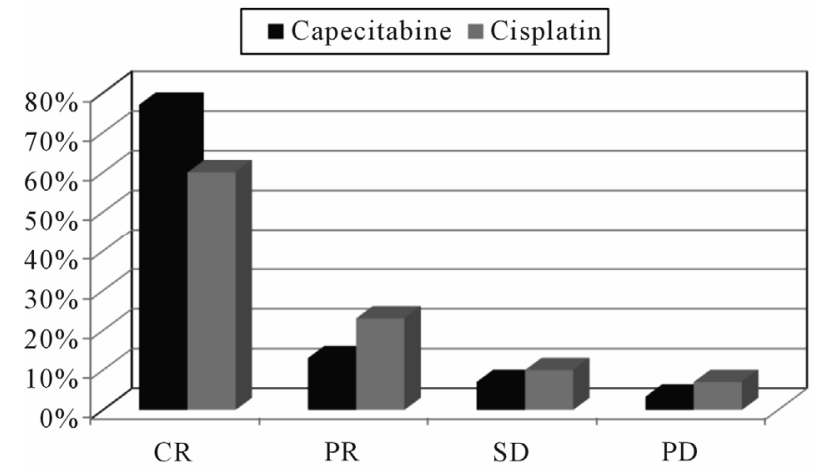

\begin{tabular}{|c|c|c|c|c|}
\hline Response & \multicolumn{2}{|c|}{ Capecitabine } & \multicolumn{2}{c|}{ Cisplatin } \\
\hline$C R$ & $23 / 30$ & $77 \%$ & $18 / 30$ & $60 \%$ \\
\hline$P R$ & $4 / 30$ & $13 \%$ & $7 / 30$ & $23 \%$ \\
\hline$S D$ & $2 / 30$ & $7 \%$ & $3 / 30$ & $10 \%$ \\
\hline$P D$ & $1 / 30$ & $3.3 \%$ & $2 / 30$ & $7 \%$ \\
\hline
\end{tabular}

Figure 1. Response rates for both groups.

Table 2. Acute toxicities in both groups.

\begin{tabular}{|c|c|c|c|c|c|c|c|c|c|}
\hline \multirow{2}{*}{$\begin{array}{l}\text { Toxicity Grade } \\
\text { \# of Cases }\end{array}$} & \multicolumn{2}{|c|}{1} & \multicolumn{2}{|c|}{2} & \multicolumn{2}{|c|}{3} & \multicolumn{2}{|c|}{4} & \multirow{2}{*}{$P$ value } \\
\hline & Capcetabine & Cisplatin & Capcetabine & Cisplatin & Capcetabine & Cisplatin & Capcetabine & Cisplatin & \\
\hline Hemoglobin & 2 & 1 & 5 & 1 & 4 & 0 & 0 & 0 & - \\
\hline White Cells & 4 & 3 & 8 & 1 & 5 & 0 & 0 & 0 & - \\
\hline Platelets & 5 & 0 & 2 & 0 & 0 & 0 & 0 & 0 & - \\
\hline Nausea & 15 & 5 & 3 & 3 & 1 & 1 & 0 & 0 & 0.011 \\
\hline Vomiting & 13 & 4 & 6 & 4 & 1 & 1 & 0 & 0 & 0.01 \\
\hline Diarrhea & 2 & 0 & 2 & 0 & 1 & 0 & 0 & 0 & - \\
\hline Palmoplantar & 0 & 0 & 2 & 0 & 1 & 0 & 0 & 0 & - \\
\hline Alopecia & 17 & 24 & 12 & 2 & 1 & 2 & 0 & 0 & 0.004 \\
\hline Skin & 8 & 20 & 17 & 6 & 2 & 1 & 0 & 0 & 0.006 \\
\hline Laryngeal edema & 10 & 8 & 5 & 2 & 2 & 1 & 0 & 0 & 0.03 \\
\hline Mucositis & 11 & 3 & 17 & 14 & 1 & 10 & 1 & 2 & 0.001 \\
\hline
\end{tabular}


cases (97\%) and grade 3 in 1 case. For the cisplatin group grade $1 / 2$ alopecia was found in 26 cases (87\%) and grade 3 in 2 cases (7\%).

Skin pigmentation and toxicity was of a higher grade in the capecitabine group ( $\mathrm{P}$ value 0.006$)$.

Laryngeal edema was more pronounced in the capecitabine group ( $\mathrm{P}$ value 0.03 )

Mucositis was more in the capecitabine group. Twenty-eight (93\%) of the patients in the capecitabine group developed grade $1 / 2$ acute radiation mucosal toxicity and 2 cases developed grade 3/4 acute mucositis. For the cisplatin group grade $1 / 2$ mucositis occurred in 17 cases (57\%) and grade 3/4 in 12 cases (40\%) (P value 0.001).

More than $10 \%$ weight loss at the end of initial treatment was experienced by 12 cases (40\%) in the capecitabine group and 10 cases (33\%) in the cisplatin group.

\section{Late Toxicity}

The incidence of late toxicity was the same for both groups. And they included neck fibrosis, trismus, chronic dysphagia with fluid diet dependence and dental carries. There prevalence was between $60 \%$ to $70 \%$ of the cases.

On analysis of survival data, the median follow-up period was $35 \pm 15$ months for overall survival and $33 \pm 10$ months for disease free survival.

The overall survival and disease-free survival rates at 2 years were $67 \%$, and $85 \%$, respectively for the capecitabine group versus $60 \%$ and $73 \%$ for the cisplatin group (Figures 2 and 3).

Performance status had a dramatic effect on overall survival with a $\mathrm{P}$ value of 0.0165 on comparing overall survival of cases with performance status 0 with those with performance status 1 .

\section{Discussion}

We believe that our work is a valuable contribution to the current search for optimal treatment for advanced head and neck cancer patients.

The use of synchronous chemo-radiotherapy has been shown in numerous randomized controlled trials, and confirmed by meta-analyses [17], to improve loco-regional control and survival.

No single agent has shown a consistent superiority in the majority of studies for chemo-radiotherapy in head and neck cancer, as highlighted in a recent review [18].

In the near and remote past intravenous 5-Fluorouracil infusion has been used in many randomized controlled trials as a single agent combined with radiotherapy in the treatment of advanced head and neck cancer [19].

The radio-sensitizing properties of 5 -FU have been
Survival Functions

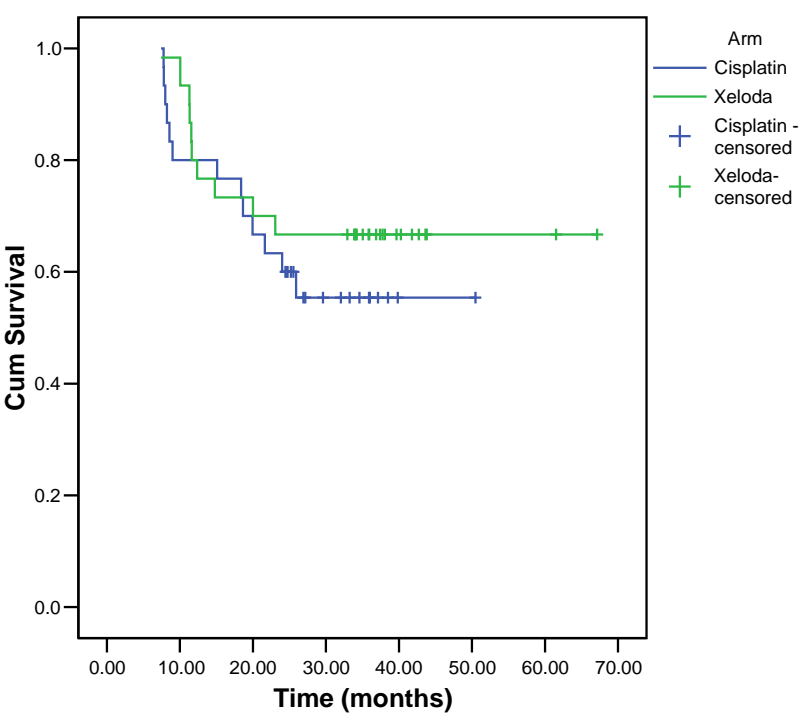

Figure 2. Overall survival for both groups.

Survival Functions

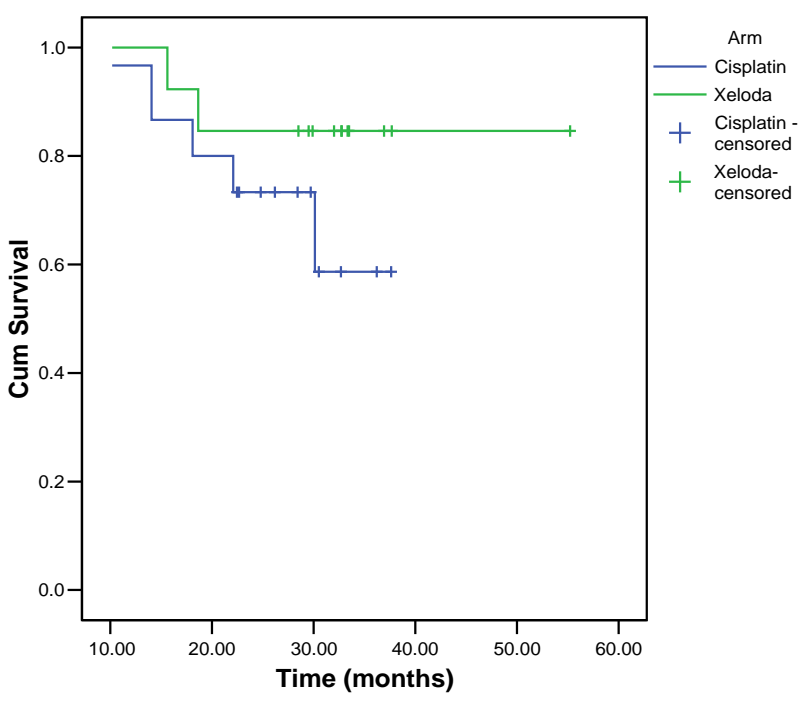

Figure 3. Disease free survival for both groups.

discovered before the sixties [20], with the well-known superiority of infusional over bolus administration shown more recently. Although not pharmacologically equivalent to infusional 5-FU, capecitabine given twice daily offers the potential of providing a prolonged exposure of tumour cells to 5-FU. Capecitabine is a non-cytotoxic pro-drug of 5-FU that is preferentially converted to 5-FU at the tumour site (a form of target therapy), exploiting the higher levels of the converting enzyme thymidine phosphorylase found in malignant cells.

When infusional 5-FU is given, the concentration of 
active drug in the tumour and normal tissue is similar. Whereas, in capecitabine we get the advantage that the expression of thymidine phosphorylase is enhanced in areas of tumour with poor perfusion, hypoxia and acidosis, a situation that exists in most advanced head and neck cancers [21]. Moreover, there is evidence that radiation leads to up-regulation of thymidine phosphorylase expression, thereby improving the efficacy of capecitabine [21]. A phase I study of capecitabine in combination with radiotherapy in head and neck cancer indicated that the maximum tolerated dose was $500 \mathrm{mg} / \mathrm{m}^{2}$ twice a day $\left(1 \mathrm{~g} / \mathrm{m}^{2}\right.$ daily) given 7 days a week, the doselimiting toxicity being exacerbation of the radiation-induced mucositis rather than systemic side-effects [15].

In the current study the complete response rate at 3 months was $77 \%$ (23/30 patients) and the partial responses were $13 \%$ (4/30), stationary disease occurred in 2 cases and progressive disease in 1 case in the capecitabine group. Whereas, the complete responses were $60 \%$ $(18 / 30)$ and the partial responses $23 \%$ (7/30), stationary disease was found in 3 cases and progressive disease in 2 cases in the cisplatin group. On analysis of survival data, the median follow-up period was $35 \pm 15$ months for overall survival and $33 \pm 10$ months for disease free survival.

The overall survival and disease-free survival rates at 2 years were $67 \%$, and $85 \%$, respectively for the capecitabine group versus $60 \%$ and $73 \%$ for the cisplatin group. These results compare very favorably and very similar with other published chemo-radiotherapy outcomes [5,22].

In our study there were no toxic deaths. There was no grade 4 bone marrow or renal toxicity, but a small risk of significant diarrhea and palmoplantar erythro-dysaesthesia was associated with capecitabine administration.

A major advantage of oral administration is the flexibility to stop medication immediately if the patient is struggling with mucositis.

Oral administration results in a reduction in inpatient stays, with improved patient convenience and reduced medical care cost.

Although 3 weekly single-agent cisplatin is the current standard for synchronous chemo-radiotherapy in SCCHN, it is important to explore other approaches [23]. Other chemotherapy options being used include weekly and daily scheduling of cisplatin [24], carboplatin [24], gemcitabine [25], capecitabine [15], as well as various combinations of cytotoxics.

In addition, capecitabine should be used with caution in patients with renal dysfunction. However, it is not contraindicated and it may be preferable to use capecitabine instead of cisplatin in patients with creatinine clearance between 30 and $60 \mathrm{ml} / \mathrm{min}$.

\section{Conclusions}

We conclude that cisplatin-based chemo-radiation is still the standard approach for the treatment of locally advanced SCCHN. Areas of active investigation are the sequential administration of induction chemotherapy followed by chemo-radiation and the integration of targeted therapies. None of the combination chemotherapy regimens demonstrated an overall survival benefit when compared to single agent methotrexate, cisplatin or 5fluorouracil.

To conclude, synchronous chemo-radiotherapy with capecitabine was found to be very effective, with excellent response, superior local control and 3-year cancerspecific survival rates. In spite of higher rates of mucosal toxicity, the treatment compliance was good due to low systemic toxicity and effective medical management of mucosal toxicity. The late toxicity is acceptable. This study is one of the innumerous studies determining the role of capecitabine in locally advanced head and neck cancer. Phase III prospective randomized controlled trials with a larger number of patients and longer follow-up will be required to confirm the role of capecitabine in the treatment of locally advanced SCCHN.

\section{REFERENCES}

[1] J. Ferlay, F. Bray, Pisani, et al., "GLOBOCAN 2002: Cancer Incidence, Mortality and Prevalence Worldwide. IARC CancerBase No. 5. Version 2.0,” IARC Press, Lyon, 2004.

[2] A. Jemal, R. Siegel and E. Ward, “Cancer Statistics,” CA: A Cancer Journal for Clinicians, Vol. 57, No. 1, 2007, pp. 43-66.

[3] J. P. Pignon, J. Bourhis, C. Domenge and L. Designe, "Chemotherapy Added to Loco-Regional Treatment for Head and Neck Squamous Cell Carcinoma: Three MetaAnalyses of Updated Individual Data. MACH-NC Collaborative Group. Meta-Analysis of Chemotherapy on Head and Neck Cancer,” Lancet, Vol. 355, 2000, pp. 949955. doi:10.1016/S0140-6736(00)90011-0

[4] J. P. Pignon, A. Le Maitre, E. Maillard and J. Bourhis, "Meta-Analysis of Chemotherapy in Head and Neck Cancer (MACH-NC): An Update on 93 Randomised Trials and 17,346 Patients,” Radiotherapy \& Oncology, Vol. 92, 2009, pp. 4-14. doi:10.1016/j.radonc.2009.04.014

[5] D. J. Adelstein, Y. Li, G. L. Adams, et al., "An Intergroup Phase III Comparison of Standard Radiation Therapy and Two Schedules of Concurrent Chemoradiotherapy in Patients with Unresectable Squamous Cell Head and Neck Cancer,” Journal of Clinical Oncology, Vol. 21, No. 20, 2003, pp. 92-98.

[6] A. S. Garden, J. Harris, E. E. Vokes, et al., "Preliminary results of Radiation Therapy Oncology Group 97-03: A Randomized Phase II Trial of Concurrent Radiation and Chemotherapy for Advanced Squamous Cell Carcinomas 
of the Head and Neck," Journal of Clinical Oncology, Vol. 22, No. 14, 2004, pp. 2856-2864.

[7] P. Sanghera, C. McConkey, K. F. Ho, et al., "Hypofractionated Accelerated Radiotherapy with Concurrent Chemotherapy for Locally Advanced Squamous Cell Carcinoma of the Head and Neck," International Journal of Radiation Oncology*Biology*Physics, Vol. 67, No. 1, 2007, pp. 342-1351.

[8] A. Trotti and S. M. Bentzen, "The Need for Adverse Effects Reporting Standards in Oncology Clinical Trials," Journal of Clinical Oncology, Vol. 22, No. 1, 2004, pp. 19-22.

[9] J. A. Bonner, P. M. Harari, J. Giralt, et al., "Radiotherapy plus Cetuximab for Squamous-Cell Carcinoma of the Head and Neck," New England Journal of Medicine, Vol. 354, No. 6, 2006, pp. 567-578.

[10] M. Peyrone, "Ueber Die Einwirkung des Ammoniaks auf Platinchlorür,” Ann Chemie Pharm, Vol. 51, No. 1, 1844, pp. 1-29. doi:10.1002/jlac.18440510102

[11] S. Trzaska, “Cisplatin,” Chemical \& Engineering News, Vol. 83, No. 25, 2005, pp. 1-162. http://pubs.acs.org/cen/coverstory/83/8325/8325cisplatin. html

[12] B. Rosenberg, L. Van Camp and T. Krigas, "Inhibition of Cell Division in Escherichia coli by Electrolysis Products from a Platinum Electrode," Nature, Vol. 205, No. 4972, 1965, pp. 698-699. doi:10.1038/205698a0

[13] D. P. Carpenter, "Reputation and Power: Organizational Image and Pharmaceutical Regulation at the FDA," Princeton University Press, Princeton, 2010.

[14] N. Sawada, T. Ishikawa, F. Sekiguchi, et al., "X-Ray Irradiation Induces Thymidine Phosphorylase and Enhances the Efficacy of Capecitabine (Xeloda) in Human Cancer Xenografts,” Clinical Cancer Research, Vol. 5, 1999, pp. 2948-2953.

[15] A. J. Sykes, N. J. Slevin, R. H. MacDougall, et al., "Results of a Phase I Study to Determine the Maximum Tolerated Dose of Capecitabine When Given Concurrently with Radical Radiotherapy in the Treatment of Squamous Cell Carcinoma of the Head and Neck,” Radiotherapy \& Oncology, Vol. 71, No. 1, 2004, pp. 81-84.

[16] C. F Lacy, L. L. Armstrong, M. P. Goldman and L. L. Lance, “Lexi-Comp’s Drug Information Handbook,” 12th Edition, Lexi-Comp Inc., 2004.
[17] J. S. Tobias, K. Monson, N. Gupta, et al., "Chemoradiotherapy for Locally Advanced Head and Neck Cancer: 10-Year Follow-Up of the UK Head and Neck (UKHAN1) Trial,” Lancet Oncology, Vol. 11, No. 1, 2010, pp. 66-74.

[18] O. Matzinger, A. Zouhair, R. O. Mirimanoff and M. Ozsahin, "Radiochemotherapy in Locally Advanced Squamous Cell Carcinomas of the Head and Neck," Clinical Oncology (Royal College of Radiologists), Vol. 21, No. 7, 2009, pp. 525-531.

[19] G. P. Browman, C. Cripps, D. I. Hodson, et al., "Placebo-Controlled Randomized Trial of Infusional Fluorouracil during Standard Radiotherapy in Locally Advanced Head and Neck Cancer," Journal of Clinical Oncology, Vol. 12, No. 12, 1994, pp. 2648-2653.

[20] M. A. Bagshaw, "Possible Role of Potentiators in Radiation Therapy," American Journal of Roentgenology, Radium Therapy, and Nuclear Medicine, Vol. 85, 1961, pp. 822-833.

[21] L. Griffiths and I. J. Stratford, "The Influence of Elevated Levels of Platelet-Derived Endothelial Cell Growth Factor/Thymidine Phosphorylase on Tumourigenicity, Tumour Growth, and Oxygenation,” International Journal of Radiation Oncology*Biology*Physics, Vol. 42, 1998, pp. 877-883.

[22] P. Huguenin, K. T. Beer, A. Allal, et al., "Concomitant Cisplatin Significantly Improves Locoregional Control in Advanced Head and Neck Cancers Treated with Hyperfractionated Radiotherapy,” Journal of Clinical Oncology, Vol. 22, No. 23, 2004, pp. 4665-4673.

[23] E. E. Cohen, M. W. Lingen and E. E. Vokes, "The Expanding Role of Systemic Therapy in Head and Neck Cancer," Journal of Clinical Oncology, Vol. 22, No. 9, 2004, pp. 1743-1752.

[24] B. Jeremic, Y. Shibamoto, B. Stanisavljevic, et al., "Radiation Therapy Alone or with Concurrent Low-Dose Daily either Cisplatin or Carboplatin in Locally Advanced Unresectable Squamous Cell Carcinoma of the Head and Neck: A Prospective Randomized Trial,” Radiotherapy \& Oncology, Vol. 43, No. 1, 1997, pp. 29-37.

[25] P. M. Specenier, W. D. van den, C. Van Laer, et al., "Phase II Feasibility Study of Concurrent Radiotherapy and Gemcitabine in Chemonaive Patients with Squamous Cell Carcinoma of the Head and Neck: Long-Term Follow Up Data,” Annals of Oncology, Vol. 18, 2007, pp. 1856-1860. doi:10.1093/annonc/mdm346 